\title{
Shaeer's Double-Eight Plication Technique for Correction of Penile Curvature
}

\section{Original Article}

\author{
Osama Shaeer
}

Department of Andrology, Faculty of Medicine, Cairo University, Egypt

\begin{abstract}
Introduction: Penile curvature can be corrected by plication of the tunica albuginea. Recurrence of curvature can be due to the suture line tearing through the tunica with repeated erection among other factors. With the suture line aligned with and parallel to the stronger outer longitudinal fibers of the tunica albuginea, those fibers are prone to splitting, with subsequent erections.

Aim of Study: The present work describes the principle behind and result of Shaeer's Double-8 plication technique for correction of penile curvature aiming at a lower recurrence rate.

Patients and Methods: Sixty patients with congenital penile curvature were randomized into the study group ( $S G, n=22)$, with curvature corrected by the "Shaeer's Double- 8 technique", and the control group (CG, $n=38)$ with curvature corrected by the 16-dot technique.

Results: Average angle of erection before correction of curvature in the SG was $39.1 \pm 8.4$, compared to $34 \pm 9$ in the CG, $\mathrm{p}=0.31$. Following correction, intra-operatively, the penis was straight in all patients. At final follow up (1-6 years (mean $3 \pm 1.2)$ ), no recurrences were observed in the SD, compared to the CG where $4 / 38$ patients $(10.5 \%)$ had a mild degree of recurrence (average angle of curvature $24.5 \pm 5.6$ degrees.)

Conclusion: Shaeer's Double-Eight Plication Technique is a simple and safe technique for correction of penile curvature, with double interlocking figure-of-eight sutures that decrease the likelihood of splitting the tougher longitudinal fibers of the tunica albuginea, with a lower recurrence rate than alternative plication techniques.
\end{abstract}

Key Words: Penile curvature, peyronie's disease, recurrence, Shaeer's Double-Eight Plication Technique, 16 dot

Received: 03 June 2017, Accepted: 14 June 2017

Corresponding Author: Osama Shaeer, MD, Department of Andrology, Faculty of Medicine, Cairo University, Egypt Tel.: +201006600606, E-mail: dr.osama@alrijal.com

ISSN: $2090-7265$

\section{INTRODUCTION}

Plication of the tunica albuginea is one of the shortening techniques employed for correction of penile curvature. It is based upon approximation of two points along the longitudinal axis of the penis. The steps classically involve initial needle entry and exit points (Fig. $1 \mathrm{a}$ and b), travel along the longitudinal axis (Fig. $1 \mathrm{c}$ ) then another needle entry and exit (Fig. $1 \mathrm{~d}$ and e). This is followed by pulling on the suture line for approximation of the two entry/exit points, then knotting.

Upon erection thereafter, the tunica albuginea composed of outer longitudinal and inner circular layers- will stretch against the resistance of the plication suture. This may be among the causes of recurrent or residual curvature where the suture may tear through the tunica albuginea. Theoretically speaking, since the plicating sutures are along and parallel to the fibres of the outer longitudinal layer of the tunica albuginea, this layer is more liable to splitting upon erection, in contrast to the inner circular layer, the fibres of which course perpendicular to the direction of the suture line. Despite the outer longitudinal layer being thicker and tougher than the inner circular, its value in maintaining the integrity of the tunica albuginea is undermined since it will offer much less resistance if the direction of force is along its fibres.

With the classical plication technique, all the suture entry/exit points are aligned along the longitudinal fibres, and it is primarily the weaker inner circular layer of the tunica albuginea that maintains the integrity against the suture line and therefore sustainability of the correction of curvature. This is less pronounced with the 16-Dot technique $^{[1]}$ since there are two parallel plication lines rather than one, though still in alignment with the fibres of the outer longitudinal layer, possibly explaining the reported recurrence rate of $15 \%^{[1]}$.

The "Double-Eight Plication Technique" (Double-8 technique) described herein avoids the fore mentioned alignment of the needle entry/exit points along the fibres of the outer longitudinal layer and distributes the tearing force on more scattered points. The longitudinal and circular 
layers will thus act together to preserve the integrity of the tunica albuginea against tearing by the plication suture with less likelihood of recurrence of curvature.

The basic principle behind the Double- 8 Technique is that each set of entry/exit points is in the form of a figureof- 8 suture. A figure- 8 suture is one where two crisscrossing tissue bites are performed (Fig. 2). This suturing technique confers higher security, sporting two tissue bites instead of one, in addition to the security due to interlocking between the two suture arms. It is useful for bringing together underlying tissues such as muscle, fascia, or extensor tendons ${ }^{[2]}$. In case of correction of curvature, it has an additional virtue since the two suture arms act across the outer longitudinal fibres rather than along them, thereby avoiding splitting.

Step-wise, the double eight plication technique comprises needle entry and exit diagonal to the longitudinal axis (Fig. $3 \mathrm{a}$ and b), then another entry and exit overlying and perpendicular to the first one, thereby forming a figure-8 (Fig. $3 \mathrm{c}$ and d). This is followed by travel (Fig. 3 e), then repetition of the fore mentioned figure- 8 (Fig. $3 \mathrm{f}$, $\mathrm{g}, \mathrm{h}$ and $\mathrm{i}$ ), then pulling on the suture line for approximation and plication; and finally knotting. It is preferable to make sure that no two needle entry/exit points are aligned along the same longitudinal line. Lack of alignment and the inherent strength and locking features of the figure- 8 sutures provide more secure plication.

However, the locking features of the two figures- $8 \mathrm{~s}$ may not allow pulling on the suture line for approximation, particularly if polyfilamentous braided sutures are used. Therefore, pulling on the suture line and approximation is performed before the last needle entry/exit as follows: The first figure-8 is performed (Fig. 3 a-d, Fig. 4 a-c), then travel (Fig. 3 e), then the first needle entry-exit of the second figure- 8 (Fig. $3 \mathrm{f}$ and g, Fig. $4 \mathrm{~d}$ and e). This is followed by pulling and approximation (Fig. $4 \mathrm{f}$ ). Approximation is maintained by a shodded haemostat applied at the exit point of the suture line to prevent separation of the approximated points (Fig. $4 \mathrm{~g}$ ). Then, with approximation maintained, the final entry/exit is performed (Fig. $4 \mathrm{~h}$ and i). The knot is tied (Fig. $4 \mathrm{j}$ ) and the haemostat removed.

Approximation is performed with the penis bent counter to the direction of curvature and the tunica albuginea dipped downwards with a haemostat such that the approximated points touch without squeezing tunica albuginea in between.

The present work describes the principle behind and result of the "Double-Eight Plication Technique" for correction of penile curvature and compares the recurrence rate to a control group operated upon by the 16-dot technique.

\section{PATIENTS AND METHODS}

Sixty patients with congenital penile curvature were included in this study. Mean age was $26.5 \pm 3.4$. All patients had normal erectile function and a normally positioned urethral meatus. Twenty two patients were randomly assigned to the study group (SG), with curvature corrected by the "Shaeer's Double- 8 technique", while 38 were randomly assigned to the control group (CG) with curvature corrected by the 16-dot technique. Curvature was ventral in 42 patients, ventro-lateral in 11 and lateral in 7. A written informed consent was obtained before surgery, as well as ethical approval of the institutional review board.

Surgery was performed under general anaesthesia. The penis was degloved through a subcoronal incision. Complete erection was induced by intra-corporal injection of saline along with basal compression. The neurovascular bundle was dissected under optical magnification for variable degrees degrees, as required for adequate access to the point of maximum curvature. Length and girth were evaluated intra-operatively in the erect state with flexible tape, with length measured from pubis to tip.

The Double- 8 technique was applied using Polyester suture material on a blunt tip needle. Approximation of the two figure- $8 \mathrm{~s}$ and knotting was performed in the semierect state with the assistant dipping the tunica albuginea between the two figrure- $8 \mathrm{~s}$ downwards and the penis bent counter to the direction of curvature (Figure 4, Video 1).

For both groups, length and girth were re-measured in the erect state following correction. The incision was closed in two layers. Patients were discharged the same day and instructed to abstain from intercourse for 4 weeks. Patients were followed up for 16- years (mean $3 \pm 1.2$ ). Recurrence rate was compared across the two groups at final follow up using patients' self-reports and self-photography in the erect state.

\section{RESULTS}

Average angle of erection before correction of curvature in the $\mathrm{SG}$ was 39.1 degrees \pm 8.4 , compared to $34 \pm 9$ in the $C G, p=0.31$. Following correction, intraoperatively, the penis was straight in all patients. Intraoperatively, changes in length and girth were comparable in both groups, with an average loss in length of $15.6 \%$ in the SD and $14.8 \%$ in the control group, $p=0.54$, and an average loss in girth of $5.4 \%$ in the SD and $3.7 \%$ in the $\mathrm{CG}, \mathrm{p}=0.87$.

Post-operatively, none of the patients reported deformity, mass formation or stitch sinus at the plication sites, apart from palpable firmness at those points. All patients reported shortening, temporary sensory loss and temporary pain. At final follow up, no recurrences were observed in the SD, compared to the CG where $438 /$ patients $(10.5 \%)$ had recurrence (average angle of curvature $24.5 \pm 5.6$ degrees.) 


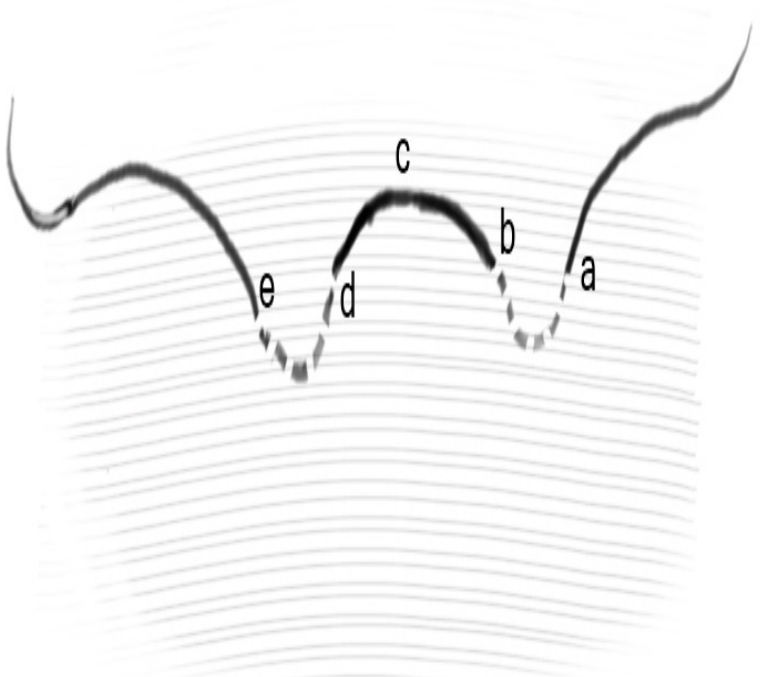

Fig. 1: Illustration of simple plication showing the suture line aligned with e outer longitudinal fibres of the tunica albuginea

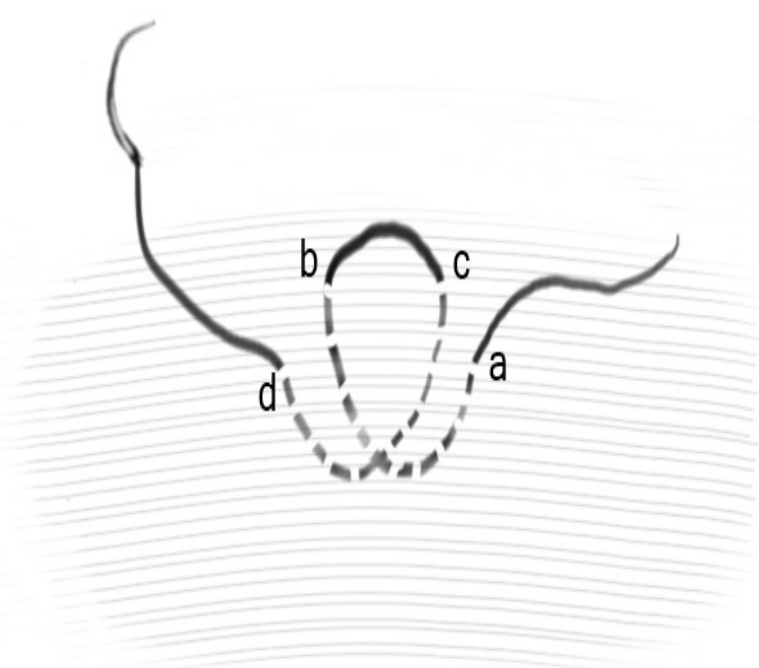

Fig. 2: Illustration of a Figure-of-8 suture

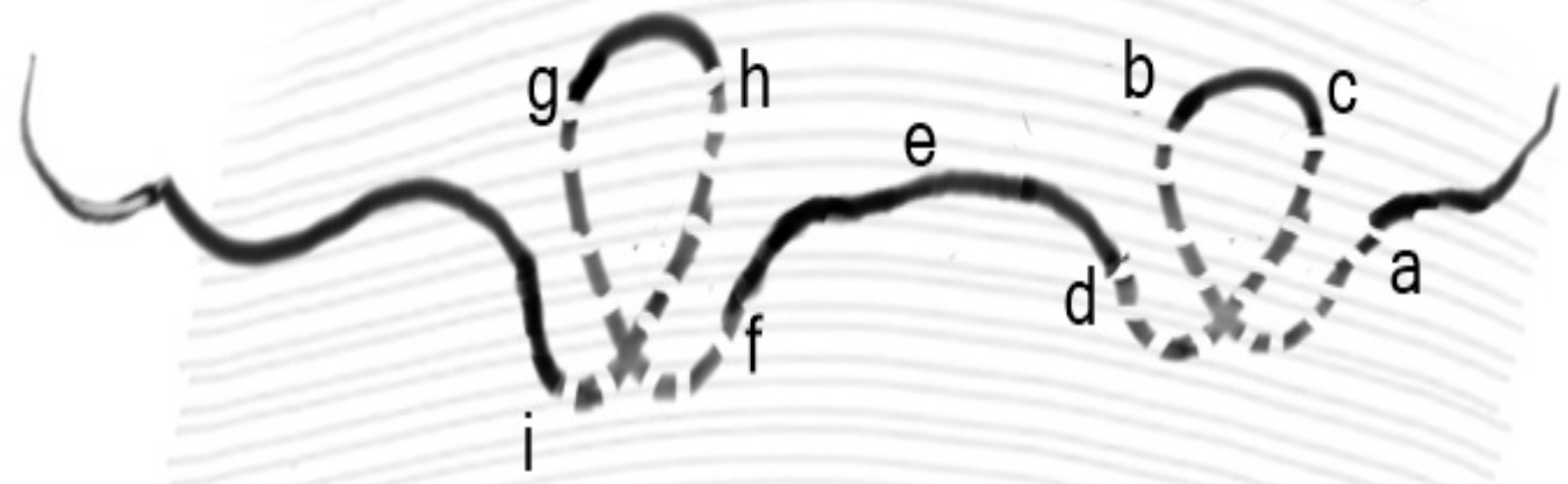

Fig. 3: Illustration of the Double-Eight Plication Technique 


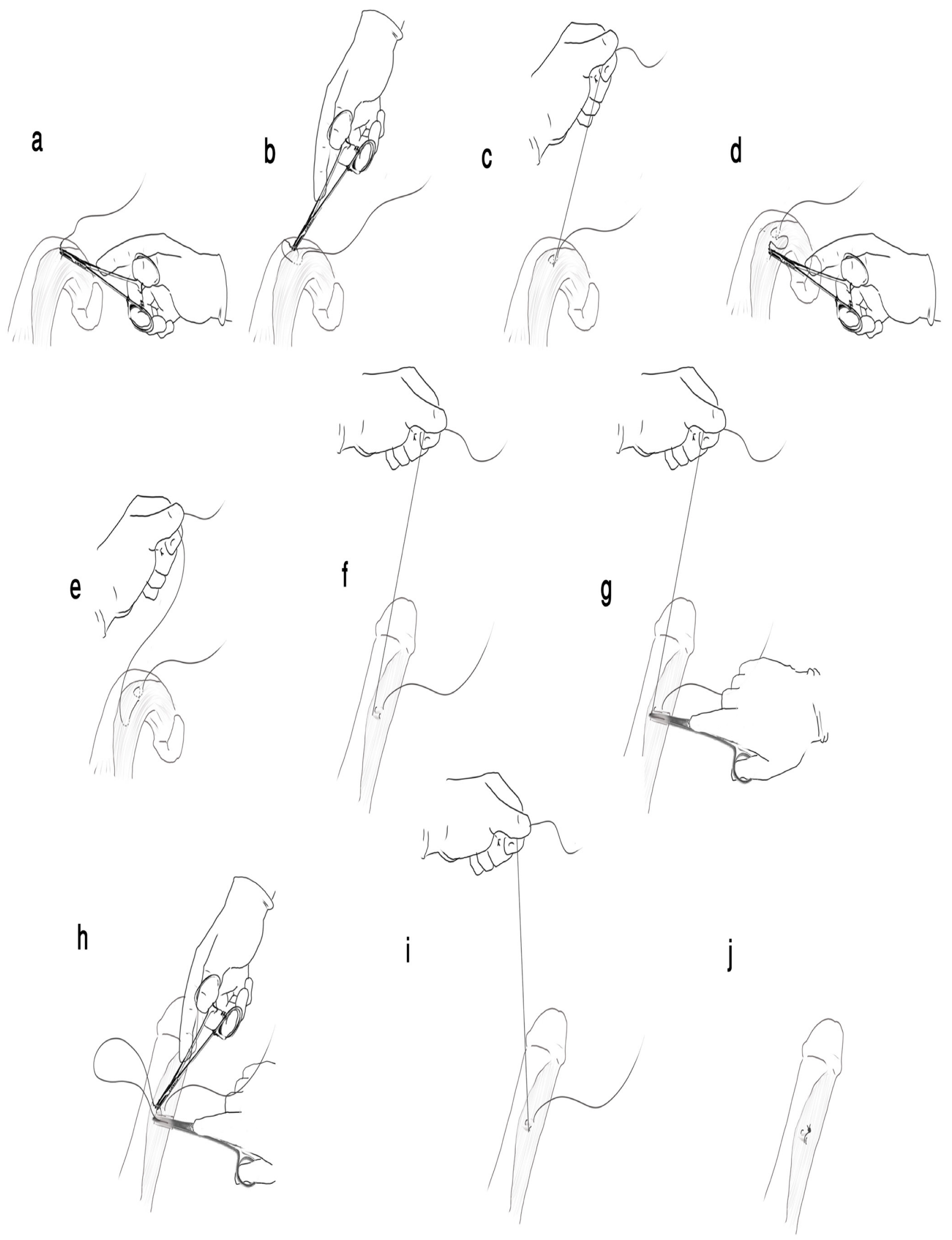

Fig. 4: Illustration of surgical steps of The Double-Eight Plication Technique 


\section{DISCUSSION}

Surgical correction for congenital curvature of the penis with a normally positioned urethral meatus is broadly according to one of four surgical principles. Tunical shortening procedures including Nesbit procedure, modified Nesbit technique, tunica albuginea plication (TAP), the 16 dot techniques, among others [1, 3-6] involve shortening of the convex side of the penis, correcting curvature on the expense of length which is reduced. Tunical lengthening procedures involving incision and grafting of the tunica albuginea on the shorter/concave side of the penis ${ }^{[7]}$ are length-preserving, though on the expense of erectile function ${ }^{[8]}$. Prosthetic surgery is reserved to patients with severe degrees of curvature or associated with refractory erectile dysfunction (ED). Finally, Shaeer's corporal rotation is a technique capable of correcting the most severe degrees of congenital curvature while preserving length and erectile function ${ }^{[9-11]}$. It is based on shifting the concavities of both corpora cavernosa from the ventral aspect of the penis to the lateral aspects in opposition, such that they flex against each other, neutralizing their curvature-inducing effect.

Focusing on tunical shortening techniques that do not involve incision of the tunica albuginea, the simple plication of the tunica albuginea is among the earliest reported ${ }^{[6]}$. Another technique is the Schroeder-Essed plication procedure involving inverted plication sutures $^{[12]}$. The 16-Dot technique involves minimal tension parallel plication sutures, with excellent durable results, avoiding the neurovascular bundles and with a minimal to no detrimental effect on erectile function.

Recurrence of curvature is possible following corrective surgery. It was reported that $15 \%$ of patients operated upon by the 16-dot procedure had recurrent curvature at a mean of 2.6 years of follow up ${ }^{[1,13]}$. Theoretically speaking, recurrence of congenital curvature corrected in adulthood after arrest of growth of the penis can be due to either the suture material tearing through the tunica albuginea with repeated erection, breaking of the suture material, poor knotting technique or necrosis of the plicated tissues due to too tight knotting.

The technique presented herein is quite simple to reproduce and adds no more operative time or suture bulk compared to alternatives, while decreasing the possibility of recurrence. What it achieves is that with every suture, the knot is attached to 8 points on different planes rather than fewer points along the same plane, thereby distributing tension and avoiding splitting of the longitudinal fibres, especially with the outer longitudinal layer being thicker and with more tensile strength compared to the inner circular, enhancing its value in protection against recurrence.

Worth mentioning is that the figure- 8 technique higher retention power on its own, that can be enhanced even more by locking the suture. This may possibly help in decreasing recurrence further, addressing the remote possibility of a loose knot.

Monofilament suture materials are known for their tensile strength and low tissue reaction. Nevertheless, they are more likely to cut through soft tissue. Polyfilamentous braided suture material are less traumatic to the tunica albuginea when stretched against the suture line upon erection, and are much less liable to form a stitch sinus.

Furthermore, cutting needles cause more tissue injury than blunt-tipped ones, paving the way for splitting the longitudinal fibres by the suture line thereafter, and increasing the likelihood of bleeding from the puncture points, hence our recommendation for blunt tips. Contrary to the general impression that the tunica albuginea is too tough for blunt tipped needles, it is our experience that this is only true in some cases of Peyronie's disease. For cases with congenital curvature, blunt tipped needles can be used without difficulty, and are less traumatic.

The Double-Eight Plication Technique was not followed by recurrence or residual curvature. Despite the benefits, recurrence is still possible for reasons other than tearing of the suture material through the tunica albuginea upon repeated erections. Other possible cause of recurrence may be breaking of the suture material.

\section{CONCLUSION}

Shaeer's Double-Eight Plication Technique is a simple and safe technique for correction of penile curvature, with double interlocking figure-of-eight sutures that decrease the likelihood of splitting the fibres of the tunica albuginea, in addition to their inherent inter-locking properties, with subsequent lower recurrence rate.

\section{CONFLICT OF INTEREST}

There are no conflicts of interest

\section{REFERENCES}

1. Gholami SS, Lue TF. Correction of penile curvature using the 16-dot plication technique: a review of 132 patients. J Urol. 2002;167(5):2066 -9. 
2. N. Semer. Practical Plastic Surgery for Nonsurgeons. Philadelphia: Hanley and Belfus; 2001. 123- p.

3. Nesbit RM. Congenital curvature of the phallus: report of three cases with description of corrective operation. 1965. J Urol. 2002;167(2 Pt 2):1187- 8; discussion 9.

4. Nesbit RM. Congenital Curvature of the Phallus: Report of Three Cases with Description of Corrective Operation. J Urol. 1965;93:230 -2.

5. Nesbit RM. Congenital Curvature of the Phallus: Report of Three Cases with Description of Corrective Operation. Trans Am Assoc Genitourin Surg. 1964;56:20 -2.

6. Baskin LS, Duckett JW. Dorsal tunica albuginea plication for hypospadias curvature. J Urol. 1994;151(6):1668- 71.

7. Devine CJ, Jr., Horton CE. Use of dermal graft to correct chordee. J Urol. 1975;113(1):56- 8.
8. Dalkin BL, Carter MF. Venogenic impotence following dermal graft repair for Peyronie's disease. J Urol. 1991;146(3):849- 51.

9. Shaeer O. Shaeer's Corporal Rotation. J Sex Med. 2010;7(1 Pt 1):16 -9.

10. Shaeer O. Shaeer's corporal rotation for lengthpreserving correction of penile curvature: modifications and 3-year experience. J Sex Med. 2008;5(11):2716- 24.

11. Shaeer O. Correction of penile curvature by rotation of the corpora cavernosa: a case report. J Sex Med. 2006;3(5):932- 7.

12. Essed E, Schroeder FH. New surgical treatment for Peyronie disease. Urology. 1985;25(6):582- 7.

13. Brant WO, Bella AJ, Lue TF. 16-Dot procedure for penile curvature. J Sex Med. 2007;4(2):277- 80 